\title{
Ciprofloxacin Cardiotoxicity and Hepatotoxicity in Humans and Animals
}

\author{
Elias Adikwu*, Nelson Brambaifa
}

Department of Pharmacology, Faculty of Basic Medical Sciences, College of Health Sciences, University of Port Harcourt, Choba Rivers State, Nigeria.

Email: *adikwuelias@gmail.com

Received January $17^{\text {th }}, 2012$; revised February $28^{\text {th }}, 2012$; accepted March $5^{\text {th }}, 2012$

\begin{abstract}
Ciprofloxacin is generally well tolerated; the most common adverse effects include gastro intestinal tract, central nervous system and hematological system effects. Recently rising cases of Ciprofloxacin associated toxicity have been reported. Experiment using animal models and clinical experience showed that Ciprofloxacin induced cardiotoxicity is marked by increase QT and QTC interval and prolonged action potential duration. This increases the risk of arrhythmia (tosarde de pointes). Ciprofloxacin induced cardiotoxic effect could be associated with blocking cardiac voltage — gated potassium channels particularly the rapid component (IKr) of the delayed rectifier potassium current. Drug interaction with inhibitors of Cytochrome P450 (CYP) mediated metabolism could be one of the underlying mechanisms. Several cases of Ciprofloxacin induced hepatoxicity have been also reported. These were characterized by extensive hepatocellular necrosis, mixed inflammatory infiltrate and abundant esinophils in the liver. Elevated liver enzymes which include serum aspartate aminotransferase, alanine aminotransferase, alkaline phosphatase, and gramma-glutamyltranferase and prolong prothrobin time were reported. The hepatotoxic effect of Ciprofloxacin as reported could be due to oxidative stress induced in the liver by Ciprofloxacin through the generation of oxidative radicals leading to depletion of protein content in hepatocytes as a consequence of nucleic acids diminution and DNA damage. This may lead to significant decrease in the number and degeneration in mitochondria which is responsible for energy supply. Conclusion: Ciprofloxacin induced cardiotoxicity and hepatotoxicity is relatively low in humans but patients' liver and cardiac function may be considered before Ciprofloxacin use.
\end{abstract}

Keywords: Ciprofloxacin; Cardiotoxicity; Hepatotoxicity; Humans; Animals

\section{Introduction}

Ciprofloxacin is a second generation fluoroquinolone with a broad spectrum of antibacterial activity. It has a good bioavailability after oral administration, good to excellent tissue penetration and is relatively safe $[1,2]$. It is very active against wide variety of pathogenic bacteria including some gram - positive and most grain-negative organism. It is used in a variety of human clinical infections like urinary tract infections, bone and soft tissue infections, respiratory tract infections, sexually transmitted infections and gastro-intestinal tract infections [3, 4].

Ciprofloxacin exerts its action by blocking bacterial DNA synthesis through inhibition of bacterial topoisomerase II (DNA gyrase) and topoisomerase IV. Inhibition of DNA gyrase prevents the relaxation of positively super coiled DNA that is required for normal transcription and replication [5-7]. Inhibition of topoisomerase IV

"Corresponding author. interferes with separation of replicated chromosomal DNA into respective daughter cells during cell division [8].

Ciprofloxacin is generally well tolerated; it remains one of the safest of all antibiotics with remarkably few reactions [9]. These reactions include gastrointestinal tract, central nervous system and hematological system effects $[10,11]$.

Recently there are some reported cases of Ciprofloxacin associated toxicological effects in humans and animals. Ciprofloxacin associated hepatotoxicity as reported by some authors manifested as hepatic failure, hepatitis, cholestatic Jaundice and acute liver injury marked by elevated level of liver enzymes [12] while Ciprofloxacin induced cardiotoxicity was reported to be associated with associated with increase QT and QTC interval, action potential prolongation, decrease heart rate and torsade de pointes [13].

Furthermore, some of these associated toxicological effects like hepatotoxicity, phototoxicity, cardiotoxicity and tendinopathy have led to the withdrawal of tema- 
floxacin, travofloxacin, grapafloxacin and sparfloxacin from US and other countries [14,15]. Despite these reports there is rising trend of Ciprofloxacin use with good therapeutic and clinical success. This work reviews reported Ciprofloxacin associated cardiotoxicity and hepatotoxicity in humans and animals.

\section{Ciprofloxacin Induced Hepatotoxicity in Humans and Animals}

Humans: Hepatotoxicity associated with Ciprofloxacin has been observed by some authors in patients treated with therapeutic doses of Ciprofloxacin. Reported Ciprofloxacin induced hepatotoxicity in most patients was characterized by elevated levels of aspartate aminotranferase (AST), alanine amino transferase (ALT), alkaline phosphate, bilirubin and leukocyte. Biopsy revealed infiltration of portal tracts by a mixed inflammatory infiltrate with permanent esinophils. Hepatocytes were markedly swollen with cell necrosis in the mid and peri-central zones [16-18].

Labowitz and Silverman (1997) [19] reported cholestatic Jaundice induced by Ciprofloxacin with no hepatomegaly or splenomegaly. Laboratory investigation revealed elevated levels of total bilirubin, direct bilirubin, alkaline phosphatase and aspartate aminotransferase. Prothrobin time was prolonged. This report agrees with other finding [20].

Cholestatic Jaundice was also repeated with repeated administration of Ciprofloxacin. In a study, $1.9 \%$ of patients taking Ciprofloxacin showed elevated SGPT levels, $1.7 \%$ had elevated SGOT levels, $0.8 \%$ had increased alkaline phosphatase levels and $0.3 \%$ had elevated bilirubin levels. Jaundice was transient and enzyme levels returned to reference range [21].

It was also observed that Ciprofloxacin induced liver injury was marked by elevated liver enzymes. Biopsy revealed extensive hepatocellular necrosis involving zones 3 and 2 of hepatic acini and a mixed inflammatory infiltration containing abundant eosinophils [22]. This report was supported by other observations [23-25]. Other cases of Ciprofloxacin induced cholestatic jaundice were also reported by other authors [26,27].

Furthermore, Ciprofloxacin induced acute cholestatic hepatitis associated with abnormal liver function test and fulminant hepatic failure marked with extensive centrilobular necrosis of the liver was also documented [28-30]. These reports agreed with reported observations by some scholars [31-33]. Due to reports associating Ciprofloxacin with hepatotoxicity it has been listed as a potential hepatotoxic agent [34].

Animals: Hepatotoxicity associated with Ciprofloxacin has been reported in experimental animal studies. Nordman et al. (1989) [35] evaluated the cytotoxicity and uptake of Ciprofloxacin in primary cultures of rats hepatocytes. They reported that Ciprofloxacin at 200 $\mathrm{mg} / \mathrm{l}$ was found to be toxic to rat hepatocytes.

This report is at variance with the report of Basaran et al. (1993) [36] who evaluated 20 and $200 \mathrm{mg} / \mathrm{kg} /$ day of Ciprofloxacin on liver function. They observed that Ciprofloxacin produced no significant histological changes in the liver. It was also observed an in-vivo genotoxicity study that Ciprofloxacin administered to rats and mice orally and subcutaneously respectively produced no hepatotoxic effects [37]. In another in-vivo study Ciprofloxacin produced significant enhancement of lipid peroxidation and alteration of glutathione redox status in hepatic tissues in rats [38].

Channa and JanJua (2003) [39] reported that Ciprofloxacin administered during gestation caused highly significant decrease in fetal liver weight. Severe liver damage, marked by dilated sinusoids, pyknotic nuclei within hepatocytes, decrease in hepatic nuclear size, decrease in hepatocytes number and few distinctly visible nucleoli hepatocytes were observed. Lymphocytic infiltration and degenerating cells were observed to be markedly increased in zone 2 and 3 in the liver of the fetus. This result agrees with the findings of Minuk et al., (1997) [40] who found that quinolones antibiotics inhibit eukaryotic as well as prokaryotic cells growth and protein synthesis by interfering with DNA and RNA replication. Similar observation was reported by other researchers. An observation by George et al., (1988) [41] supported the above reports. They found that in Ciprofloxacin treated liver there was marked congestion of sinusoids with midzonal and centrilobular necrosis and leucocytic infiltration.

Nadia, 2006 [42] reported that administration of therapeutic and double therapeutic doses of Ciprofloxacin (57 and $114 \mathrm{mg} / \mathrm{kg}$ ) in two periods (pre implantation and post implantation of pregnancy) induced various changes in liver of pregnant rats and their fetuses. These changes varied from dilatation of hepatic portal vein and sinusoids, increase in Kupffer and inflammatory cells, degenerative alterations and massive number of lymphoid cells aggregation in the portal area. Degeneration progressed to necrosis and pyknotic nuclei, and focal fibrosis. In addition, the liver of the fetuses showed fatty changes, haemolysis of the blood in sinusoidal spaces and severe dilatation of central vein with focal hemosiderosis, necrosis and pyknotic nuclei.

On the other hand, contradictory results have reported the safety of Ciprofloxacin on the hepatic system. For instance, Keita et al. (1998) [43] found that Ciprofloxacin administered at a dose of $100 \mathrm{mg} / \mathrm{kg}$, improved survival rates and hepatic regenerative activity in a rat model with hepatic failure. Minuk et al. (1995) [44] and Zhang et al. (1996) [45] reported that Ciprofloxacin reversed the inhibitory effects in ethanol and carbon tetra- 
chloride induced models of hepatic injury.

Mechanism: The mechanism by which Ciprofloxacin induced hepatotoxicity is not well understood. Some researchers suggested that free oxidative radical generation could induce oxidative stress in the liver leading to organ damage. This is supported by reported increased in lipid hydroperoxide (LOOH) in the liver of mice by Ciprofloxacin which is a marker of induced oxidative stress in the liver. Oxidative stress induced by Ciprofloxacin in liver of mouse was decrease by ascorbic acid probably by inhibiting or decreasing oxidative radical generation by Ciprofloxacin in the liver [46].

Gurbay et al. (2001) [47] reported that a six-line 4-POBN/radicals adduct was detected when rat hepatic microsomes were incubated in various concentrations of Ciprofloxacin at different time intervals in the presence of 4-POBN and NADPH.

The formation of free radicals by Ciprofloxacin in the microsomal system might provide an explanation for the mechanism of adverse effects observed after administration of this drug to patients. The mechanism of radical formation by Ciprofloxacin might be a result of metabolism of this drug by Cytochrome P450 and/or redox reaction. It was reported that the preferential zone-3 distribution of hepatic damage, suggests a possible involvement of the Cytochrome P450 enzyme. This enzyme activity is highest in zone-3, and it has been shown that Ciprofloxacin suppresses relevant Cytochrome P450 at the transcription level [48].

Furthermore the generation of oxidative radicals by Ciprofloxacin may lead to depletion of protein content in hepatocytes which is a consequence of nucleic acids diminution and the damage of DNA. This may lead to significant decrease in the number and degeneration in mitochondria which is responsible for energy supply [49]. This statement is further supported by the fact that Ciprofloxacin antibacterial activity has been ascribed to DNA binding, resulting in a marked inhibition of bacterial DNA topoisomerase [50-52].

Ciprofloxacin is still an effective and safe antibiotic, however, clinicians should be careful about Ciprofloxacin related hepatic injury, the consequences can be severe. Patients' drug history and liver function test may be required before Ciprofloxacin use.

\section{Ciprofloxacin Induced Cardiotoxicity in Humans and Animals}

Animals: Experiments with animals have shown that Ciprofloxacin has the potential to induce cardiotoxic effect. One of these experiments showed that Ciprofloxacin induced changes in electrocardiogram (ECG) in guinea pigs [53]. Ciprofloxacin induced cardiotoxicity was reported to be marked by increase in biomarkers of cardiac function [54].

In myocardiac evaluation of Ciprofloxacin in Juvenile rat and the role of oxidative stress in heart toxicity, 25 and $50 \mathrm{mg} / \mathrm{kg}$ of Ciprofloxacin was administered to rats for 1 week and biochemical parameters, including malondialdehyde and nitric oxide were evaluated. Result showed that Ciprofloxacin might have caused myocardiotoxicity in these rats by inducing oxidative stress in the heart and nitric oxide may be partly responsible for this toxicity [55].

This is supported by a study conducted with an anaesthetized rabbits in which Ciprofloxacin $(1-30 \mathrm{mg} / \mathrm{kg})$ caused a transient dose-related decrease in heart rate, but after a dose ten times higher $(300 \mathrm{mg} / \mathrm{kg})$ ventricular tachycardia and arrhythmias were also observed. Although weak evidence linked Ciprofloxacin with QT mediated arrhythmias [56]. It was also reported that administration of $(20 \mathrm{mg} / \mathrm{kg})$ intravenously in rats significantly increased QT and QTC intervals [57].

Some recent studies have demonstrated comparative risk of Ciprofloxacin with other fluoroquinolones in guinea pig isolated right ventricular myocyte model. It was observed that Ciprofloxacin and Levofloxacin similarly prolonged action potential duration by $0.6 \%-3.3 \%$ [58]. This agrees with the finding of Partmore et al., (2000) [59] who reported that Ciprofloxacin significantly prolong action potential duration in left and right ventricular purkinje fibres isolated from canine hearts in a concentration dependant manner. This is further supported by the ability of Ciprofloxacin to significantly induce QT interval and action potential prolongation with early after depolarization and torsade de pointes effect [60].

The effect of intravenous $(10 \mathrm{mg} / \mathrm{kg}$ ) of Ciprofloxacin on the electrocardiogram of healthy dogs was evaluated, insignificant changes were observed in QT, QTC, PR, and QRS interval, heart rate, ST segment and $\mathrm{T}$ wave amplitude. Despite the occurrence of ECG changes following intravenous Ciprofloxacin administration neither dangerous rhythm disturbances nor serious ECG changes were seen in this study [61].

Humans: The cardiotoxic potential of Ciprofloxacin has been reported from clinical experience. In a more recent report Prabhakar and Krahn (2004) [62] recorded marked QT prolongation with recurrent syncope and documented tosarde de pointes requiring defibrillation in two female patients following Ciprofloxacin administration. In both cases the QT normalized after cessation of Ciprofloxacin. Ciprofloxacin has also been linked to some cases of serious QT interval lengthening and tosarde de pointes [63].

This is at variance with some observations. In a prospective evaluation of 38 patients, 23 women and 15 men who received either Ciprofloxacin or Levofloxacin, it was observed that neither Levofloxacin nor Ciprofloxacin 
significantly prolong QTC internal over baseline [64].

Knoir et al., (2008) [65] reported that a pediatric patient with Crohns disease and colitis developed a prolonged QT internal and slow heart rate within 48 hours of treatment with intravenous Ciprofloxacin $400 \mathrm{mg}$ twice daily. QT internal returns to normal when Ciprofloxacin was stopped.

This agrees with the findings of Nair et al., (2008) [66] who reported that a patient receiving methadone treatment developed prolonged QT interval and torsade de pointes following the addition of Ciprofloxacin. Methadone is also known to be associated with tosarde de pointes.

Another case of prolonged QT internal and tosarde de pointes due to Ciprofloxacin was also reported, which was marked by polymorphic ventricular tachycardia and long QT (QTC $596 \mathrm{~ms})$. After 72 hours of withdrawal of Ciprofloxacin, the disappearance of changes in cardiac repolarization was noted [67]. This agrees with the report of Flanagan et al., (2006) [68] who observed tosarde de pointes, polymorphic ventricular tachycardiac associated with QT internal prolongation caused by intravenous Ciprofloxacin given for pneumonia in a 22-year old healthy marine.

A patient was diagnosed of tosarde de pointes which electrocardiogram revealed severely prolonged QT internal; it resolved when the administered Ciprofloxacin was withdrawn [69]. A case of Ciprofloxacin induced bradycardiac was also reported [70]. Konstantinos et al., (2006) [71] reported a case of QT interval prolongation after Ciprofloxacin administration in a patient receiving olanzapine. A similar case of QT prolongation was also reported when Ciprofloxacin was administered with azimilide in a patient with implanted cardioverter defibrillator [72]. These reports agreed with some observations [73].

Ciprofloxacin was reported to induced cardiac arrest by QT prolongation $(\mathrm{QTC}=0.62)$ within 24 hours with documented syncope. The patient was under amiodarone and sotalol therapy for atrial fibrillation with no obvious QT prolongation prior to Ciprofloxacin therapy [74]. This is at variance with the findings of Noel et al., (2003) [75] who posited that Ciprofloxacin produced insignificant increase in QT and QTC interval when a single dose of $500 \mathrm{mg}$ was administered to healthy adult volunteers.

Furthermore it was reported that quinolones block the rapid component of delayed rectifier potassium current (IK) in a dose dependent manner. This electrophysiological action translates into prolongation of the QT interval and may cause the development of torsade de pointes. QT prolongation appears to be a class issue with Ciprofloxacin as the safest base on available evidence [76].

Cases of Ciprofloxacin induced QT prolongation and tosarde de pointes in human have been rarely reported. This shows that in human Ciprofloxacin may not be cardiotoxic.

Mechanism: The mechanism of Ciprofloxacin induced cardiotoxicity is not well understood. Some authors said fluoroquinolones induced cardiotoxicity could be associated with blocking cardiac voltage - gated potassium channels particularly the rapid component (IKr) of the delayed rectifier potassium current. In vitro studies showed that fluoroquinolones block HERG (the human ether-ago-go-gene) responsible for the $\mathrm{IKr}$ and subsequently prolong QT and Torsade de pointes [77,78].

Drug interaction with inhibitors of Cytochrome P450 (CYP) mediated metabolism may be one of the underlying mechanisms. It has been shown that the higher the dose of fluoroquinolone and serum concentration the higher the QT prolongation risk and subsequently the risk of tosarde de pointes [79].

Furthermore it has been reported that radical in position 5 of the fluoroquinolone ring is responsible for QTC prolongation. A proton $(\mathrm{H})$ at this position has been reported to be associated with QT prolongation in Ciprofloxacin [80].

This review shows that Ciprofloxacin may have cardiotoxic potential. Patients cardiac function status may be considered before Ciprofloxacin clinical use.

\section{REFERENCES}

[1] P. Ball and G. Tillotson, "Tolerability of Fluoroquinolones Antibiotics Past Present and Future," Drug Safe, Vol. 13, 1995, pp. 345-358. doi:10.2165/00002018-199513060-00004

[2] M. Papich, "Antibacterial Drug Therapy Focus on New Drugs," Clinical Pharmacology \& Therapeutics, Vol. 28, 1998, pp. 215-231.

[3] B. Sub and B. Lorber, "Quinolones," Medicina Clínica, Vol. 79, 1995, pp. 869-894.

[4] D. C. Hooper and J. S. Wolfson, "The Fluoroquinolones: Pharmacology, Clinical Uses Toxicities in Human," Antimicrobial Agents and Chemotherapy, Vol. 28, No. 5, 1985, pp. 716-721.

[5] K. Lica and X. Zhao, "DNA Gyrase Topoisomerase IV and the 4-Quinolones," Microbiology and Molecular Biology Reviews, Vol. 61, No. 3, 1997, pp. 377-392.

[6] D C. Hooper, "Quinolones," In: G. L. Mandell, J. E. Bennett and R. Dolin, Eds., Mandell Douglas and Bennett's Principles and Practice of Infections Disease, 5th Edition, Churchill Livingstone, New York, 2000, pp. 403423.

[7] F. L. Alovero, X. S. Pan, J. E. Morris, R. H. Manzo and L. M. Fisher, "Engineering the Specificity of Antibacterial Fluoroquinolones, Benzene Sulfonamide Modifications at C-7 of Ciprofloxacin Change Its Primary Target in Streptococcus Pneumoniae from Topoisomerase IV to Gyrase," Antimicrobial Agents and Chemotherapy, Vol. 44, 
No. 2, 2000, pp. 320-325. doi:10.1128/AAC.44.2.320-325.2000

[8] L. A. Mitscher and Z. Mao, "Structure Activity Relationships of Quinolones in Fluoroquinolones Antibiotics," In: A. R. Ronald and D. E. Low, Eds., Birkhauser Based 2003, pp. 11-48.

[9] P. Ball, L. Mandell, Y. Niki and G. Tillotson, "Comparative Tolerability of the Newer Fluoroquinolone Antimicrobials," Drug Safety, Vol. 21, 1999, pp. 407-421. doi:10.2165/00002018-199921050-00005

[10] W. A. Petri, "Sulfonamides, Trimethoprim, Sulfomethoxazole, Quinolones and Agents of Urinary Tract Infections," In: Brunton L. L., Ed., Goodman \& Gilman's the Pharmacological Basics of therapeutics, 12th Edition, MC Graw Hill Companies Inc., New York, 2001, pp. 1463-1476.

[11] H. Lode, K. Bomer and P. Koeppe, "Pharmacodynamics of Fluoroquinolones Clin," Clinical Infectious Disease, Vol. 27, 1998, pp. 33-39. doi:10.1086/514623

[12] M. A. Contreras, R. Luna, J. Mulero and J. L. Andreu, "Severe Ciprofloxacin Induced Acute Hepatitis," European Journal of Clinical Microbiology \& Infectious Diseases, Vol. 20, No. 6, 2001, pp. 434-435. doi:10.1007/s100960100513

[13] M. E. Falagas, P. I. Rafailidis and E. S. Rosmarakis, "Arrhythmias Associated with Fluoroquinolone Therapy," International Journal of Antimicrobial Agents, Vol. 29, No. 4, 2007, pp. 374-379.

doi:10.1016/j.ijantimicag.2006.11.011

[14] Otsuka Pharmaceutical Company Limited, "Withdrawal of Approval of a New Drug application: Correction," Federal Register, Vol. 72, No. 130, 2007, Article ID: 32852

[15] Adverse Effects of Fluoroquinolones, Wikipedia the Free Encyclopedia.

[16] M. K. Bhagirath, "A Case Report of Highly Suspected Ciprofloxacin-Induced Hepatotoxicity," Turkish Journal of Gastroenterology, Vol. 19, No. 3, 2008, pp. 204-206

[17] J. P. Villeneuve, C. Davies and J. Cole, "Suspected Ciprofloxacin-Induced Hepatotoxicity," Annals of Pharmacotherapy, Vol. 29, 1995, pp. 294-296

[18] A. C. Hirsch and L. M. Lundquist, "Ciprofloxacin-Induce Hepatotoxicity Resolved with Levofloxacin: A Case Report and a Review of the Literature," Hospital Pharmacy, Vol. 44, No. 11, 2009, pp. 978-983.

doi:10.1310/hpj4411-978

[19] J. K. Labowitz and W. B. Silverman, "Cholestatic Jaundice Induced by Ciprofloxacin," Digestive Diseases and Sciences, Vol. 42, No. 1, 1997, pp. 192-194. doi:10.1023/A:1018870029216

[20] A. Aggarwal and J. Gurka, "Probable Ciprofloxacin Induced Cholestasis," Australian and New Zealand Journal of Medicine, Vol. 25, No. 5, 1995, pp. 541-542. doi:10.1111/j.1445-5994.1995.tb01506.x

[21] N. M. Bass. "Drug Induced Liver Disease in Current Diagnosis and Treatment," In: S. Friedman, K. Mcqauid and J. Grendell, Eds., Gastroenterology, 2nd Edition, McGraw-Hill Professional, New York, 2003, pp. 664-679
[22] A. Zimpfer, A. Propst, G. Mikuz, W. Vogel, L. Terracciano and S. Stadmann, "Ciprofloxacin-Induced Acute Liver Injury: Case Report and Review of Literature," Virchows Arch, Vol. 444, No. 1, 2004, pp. 87-89. doi:10.1007/s00428-003-0917-9

[23] A. J. Dichiara, A. Z. Goodman and H. E. Sherman, "Ciprofloxacin-Induced Acute Cholestatic Liver Injury and Associated Renal Failure a Case Report and Review," Minerva Gastroenterologica E Dietologica, Vol. 54, No. 3, 2008, pp. 307-315.

[24] S. E. Orman, H. S. Conjevaram, R. Vuppalachi, W. T. Freston, J. Rochen, E. D. Kleiner, P. H. Hayashi, "Clinical and Histopathologic Features of Fluoroquinolone-Induce Liver Injury," Clinical Gastroenterology and Hepatology, Vol. 3, 2001, pp. 517-523.

[25] Z. M. Goetz and P. R. Galle, "Schwarting. Non Fatal Acute Liver Injury Possibly Related to High-Dose Ciprofloxacin," European Journal of Clinical Microbiology \& Infectious Diseases, Vol. 22, No. 5, 2003, pp. 294-296.

[26] O. Sherman and J. L. Beizer, "Possible Ciprofloxacin Induce Acute Cholestatic Jaundice," Annals of Pharmacotherapy, Vol. 28, No. 10, 1994, pp. 1162-1164.

[27] J. R. Levinson and A. Kumar, "Ciprofloxacin-Induced Cholestatic Jaundice: A Case Report (Abstract)," American Journal of Gastroenterology, Vol. 88, 1993, p. 1619.

[28] S. Fuchs, Z. Simeon and M. Brezis, "Fatal Hepatic Failure Associated with Ciprofloxacin," Lancet, Vol. 19, No. 343, 1994, pp. 738-739. doi:10.1016/S0140-6736(94)91624-1

[29] B. K. Grassmick, V. T. Lehr and A. S. Sundareson, "Fulminant Hepatic Failure Possibly Related to Ciprofloxacin," Annals of Pharmacotherapy, Vol. 26, No. 5, 1992, pp. 636-639.

[30] M. Pfeiffer, C. Reiter, S. Fuchs, Z. Simon and M. Brezis, "Fatal Hepatic Failure Associated with Ciprofloxacin," Lancet, Vol. 343, No. 8899, 1994, pp. 738-739. doi:10.1016/S0140-6736(94)91624-1

[31] M. Alcalde, M. S. Donoso, M. Carcfa-Diaz and J. M. Puscasio, "Liver Dysfunction Due to Ciprofloxacin," Acta Gastro-Enterologica Belgica, Vol. 58, 1995, pp. 475-476.

[32] E. Cholongitas, C. Georgousaki, S. Spyrou and M. Deisenaki. "Ciprofloxacin Induce Acute Cholestatic Hepatitis," Annals of Hepatology, Vol. 83, No. 4, 2009, pp. 400-401.

[33] B. S. Thakur, A. K. Jain, S. Sirkar, G. Joshi and R. Joshi. "Ciprofloxacin Induced Cholestatic Jaundice," Indian Society of Gastroenterology, Vol. 26, No. 1, 2007, pp. 5152.

[34] T. Vial, M. Biour, J. Descotes and C. Trepo, "Antibiotics Associated Hepatitis, Update from 1990," Annals of Pharmacotherapy, Vol. 31, No. 2, 1997, pp. 204-220

[35] P. Nordmann, A. Pechinot and A. Kazmierczak, "Cytotoxicity and Uptake of Perfloxacin, Ciprofloxacin and Ofloxacin in Primary Culture of Rat Hepatocytes," Journal of Antimicrobial Chemotherapy, Vol. 24, No. 3, 1981, pp. 355-365. doi:10.1093/jac/24.3.355

[36] A. Basaran, N. Basaran, H. V. Gunes, E. Acikalin, P. G. Timural, I. Degirmenci, E. A. Cakmak and A. G. Tomati, 
"Effect of Ciprofloxacin on Chromosomes, and Hepatic and Renal Functions in Rats," Chemotherapy, Vol. 39, No. 3, 1993, pp. 182-188. doi:10.1159/000239124

[37] B A. Herbold, S. Y. Brendler-Schwaab and H. J. Ahr, "Ciprofloxacin: In Vivo Genotoxicity Studies," Mutation Research, Vol. 498, 2001, pp. 193-205.

[38] F. Hincal and T. Taskin, "The Mechanism Induced by Ciprofloxacin May Involve the Generation of Free Radicals and the Activation of Excitatory Amino Acid Receptors," Abstracts of the International Congress of Toxicology, W. A. Abstr 99, VII Seattle, 1995, p. 27.

[39] M. A. Channa and M. Z. Janjua, "Effect of Ciprofloxacin on Fetal Hepatocytes," Journal of the Pakistan Medical Association, Vol. 53, No. 10, 2003, pp. 448-450.

[40] G. Minuk, N. Assy and L. Ding, "Effects of Quinolone Antibiotic on Hepatic Growth and Protein Synthesis Following Partial Hepatectomy in Rats," Journal of Gastroenterology and Hepatology, Vol. 12, No. 1, 1997, pp. 5-7. doi:10.1111/j.1440-1746.1997.tb00346.x

[41] A. I. Weyers, L. I. Ugma, O. H. Carcia and G. B. Gloria, "Ciprofloxacin Increases Hepatic and Renal Lipid Hydroperoxide Levels in Mice," Biocell, Vol. 26, No. 2, 2002, pp. 225-228.

[42] H. I. Nadia, "Assessment of Histopathological and Histological Changes in Liver of Pregnant Female Rats Their Fetuses Following Ciprofloxacin Administration," Journal of Egypt Society of Toxicology, Vol. 35, 2006, pp. 7-17.

[43] D. E. Keita, K. N. Assy, T. Gauther, M. Zhang, F. Adrienne, A. Meyers and Y. M. Gerald, "The Beneficial Effects of Ciprofloxacin on Survival and Hepatic Regenerative Activity in a Rat Model of Fulminant Hepatic Failure," Hepatology, Vol. 27, No. 2, 1998, pp. 533-536. doi:10.1002/hep.510270230

[44] G. Minuk, T. Gauthier, X. Zhang, G. Wang and F. Burczynski, "Ciprofloxacin Reverses the Inhibitory Effect of Acute Ethanol Exposure on Hepatic Regeneration in Rat," Hepatology, Vol. 22, 1995, pp. 1797-1800.

[45] M. Zhang, G. Song and G. Minuk, "The Effects of Hepatic Stimulator Substance Chinese Herbal Medicine Selenium Vitamin E and Ciprofloxacin on Hepatic Fibrosis in Rats," Gastroenterology, Vol. 110, No. 4, 2007, pp. 1150-1155. doi:10.1053/gast.1996.v110.pm8613004

[46] I. U. Weyers, G. O. Hugo and B. G. Nora, "Ciprofloxacin Increases Hepatic and Renal Lipid Hydroperoxide Levels in Mice," Biocell, Vol. 26, No. 2, 2002, pp. 225-228.

[47] A. Gurbay, B. Gonthier, O. Daveloose, A. Favier and F. Hincal, "Microsomal Metabolism of Ciprofloxacin Generates Free Radicals," Free Radical Biology and Medicine, Vol. 30, No. 10, 2001, pp. 1118-1121.

[48] H. J. Xie, G. L. Bromberg, S. C. Lundgren, P. C. Meurling and A. H. M. Rane, "Alteration of Pharmacokinetics of Cyclophosphamide and Suppression of the Cytochrome P450 Genes by Ciprofloxacin," Bone Marrow Transplant, Vol. 31, 2003, pp. 197-203. doi:10.1038/sj.bmt.1703815

[49] A. Pino, A. V. Maura and L. Masciangelo, "Evaluation of DNA Damage Induced by Norfloxacin in Liver and Kid- ney of Adult Rats and Fetal Tissues after Transplacental Exposure," Mutation Research, Vol. 264, 1991, pp. 81-85. doi:10.1016/0165-7992(91)90049-A

[50] A. G. Gilman, T. N. Rall, A. S. Nies and P. Taylor, "The Pharmacological Basis of Therapeutics," 8th Edition, Pergamon, New York, 1990, pp. 1057-1060.

[51] G. C. Crumplin, M. Kenwright and T. Hirst, "Investigation into the Mechanism of Action of Antibacterial Agent Norfloxacin," Journal of Antimicrobial Chemotherapy, Vol. 13, No. 1B, 1984, pp. 9-23.

[52] M. Gellert, "DNA Topoisomerase," Annual Review of Biochemistry, Vol. 50, 1981, pp. 879-910. doi:10.1146/annurev.bi.50.070181.004311

[53] X. L. Kang, Z. Liming, L. Liming and Y.-S. Wang, "Effects of Quinolones on Electrocardiogram in Guinea Pigs," Chinese Journal of Antibiotics, 2004, pp. 7-12.

[54] K. Pispirogos and K. Chrysanthopoulos, "Evaluation of Cardio Subacute Toxicity of Ciprofloxacin in Rats Using Serum Biochemical Parameters," Arzneimittel Forschung, Vol. 51, No.7, 2001, pp. 582-587.

[55] A. Saracoglu, H. E. Temel, B. Ergun and O. Colak, "Oxidative Stress Medicated Myocardiotoxicity of Ciprofloxacin and Ofloxacin in Juvenile Rats," Drug Chemical Toxicology, Vol. 32, No. 3, 2009, pp. 238-242.

[56] R. Frothingham, "Rates of Torsades de Pointes Associated with Ciprofloxacin, Ofloxacin, Levofloxacin, Gatifloxacin and Moxifloxacin," Pharmacotherapy, Vol. 21, No. 12, 2001, pp. 1468-1472. doi:10.1592/phco.21.20.1468.34482

[57] Y. M. Daba, A. M. Fouda and M. G. Dahab, "Interactions of Metochlorpramide and Ciprofloxacin on Electrocardiographic Indices in Anesthetized Normal and Hyperthemic Rats," Saudi Pharmaceutical Journal, Vol. 14, No. 1, 2006.

[58] J. B. Kahn, "Quinolone-Induced QT Interval Prolongation a Not So Unexpected Class Effect," Journal of Antimicrobial Chemotherapy, Vol. 46, No. 5, 2000, pp. 847848. doi:10.1093/jac/46.5.847

[59] L. Patmore, S. Fraser, D. Mair and A. Templeton, "Effects of Sparfloxacin, Grapafloxacin, Moxifloxacin and Ciprofloxacin on Cardiac Action Potential Duration," European Journal of Pharmacology, Vol. 406, No. 3, 2000, pp. 449-452. doi:10.1016/S0014-2999(00)00694-4

[60] P. Milberg, E. Hilker, S. Ramtein, Y. Cakir, J. Stypmann and E. Marcus, "Proarrhythmia as a Class Effect of Quinolone: Increased Dispersion of Repolarization and Triangulation of Action Potential Predict Torsade de Pointes," Journal of Cardiovascular Electrophysiology, Vol. 18, No. 7, 2007, pp. 1-8.

[61] M. S. Ghaffari and R. Parsamehr, "The Effect of Intravenous Ciprofloxacin on the Electrocardiogram of Healthy Dogs," Veterinary Research Communications, Vol. 33, No. 8, 2009, pp. 987-990. doi:10.1007/s11259-009-9317-1

[62] M. Prabhakar and A. D. Krahn, "Ciprofloxacin Induced Acquired Long QT Syndrome," Heart Rhythm, Vol. 1, No. 5, 2001, pp. 624-626. doi:10.1016/i.hrthm.2004.06.020 
[63] S. K. Daya, R. M Gowda and I. A. Khan, "Ciprofloxacin and Hypoglycemia-Induced Torsade de Pointes Triggered by Hemodialysis," American Journal of Therapeutics, Vol. 11, No. 1, 2004, pp. 77-79.

doi:10.1097/00045391-200401000-00014

[64] A. N. Makaryus, K. Byrns, M. N. Makaryus, U. Natarajan, C. Singer and B. Goldner, "Effect of Ciprofloxacin and Levofloxacin on the QT Interval: Is This a Significant Clinical Event," Southern Medical Journal, Vol. 99, No. 1, 2006, pp. 52-56. doi:10.1097/01.smj.0000197124.31174.7e

[65] J. P. Knoir, M. Moshfeghi and M. C. Sokoloski, "Ciprofloxacin-Induced Q-T Interval Prolongation," American Journal of Health System Pharmacy, Vol. 65, No. 6, 2008, pp. 547-551. doi:10.2146/ajhp070081

[66] M. K. Nair, K. Patel and P. J. Starer, "CiprofloxacinInduced Torsade de Pointes in a Methadone-Dependent Patient," Addiction, Vol. 103, No. 12, 2008, pp. 20622064. doi:10.1111/j.1360-0443.2008.02390.x

[67] J. S. Arce, R. Romeo and P. Solórzano, "A Case of Prolonged QT Interval and Torsade de Pointes Due to Ciprofloxacin," Revista Espanola de Cardiologia, Vol. 63, No. 1, 2010, pp. 111-125. doi:10.1016/S1885-5857(10)70017-7

[68] C. M. Flanagan, E. S. Mitchell and M. Haigney, "Ciprofloxacin Induced Torsade de Pointes," International Journal of Cardiology, Vol. 113, No. 2, 2006, pp. 239-241. doi:10.1016/j.ijcard.2005.08.048

[69] M. Ibrahim and B. Omar, "Ciprofloxacin Induce Torsade de Pointes," American Journal of Emergency Medicine, Vol. 30, No. 252, 2012, pp. 5-9.

[70] C. N. Dhobi and T. A. Shafiga, "Ciprofloxacin Induced Bradycardiac: Case Report,” Letter, Vol. 26, No. 2, 1994, p. 164.

[71] P. L. Konstantino, S. Antonios, P. K. Stavios, E. Michalis, K. Panagiotis and F. Kadaras, "Drug Induced QT Interval Prolongation after Ciprofloxacin Administration in a Patient Receiving Olanzapine," International Journal of
Cardiology, Vol. 109, No. 2, 2006, pp. 273-274. doi:10.1016/j.ijcard.2005.04.031

[72] J. Kazmierczak, M. P. Pogorzelska and R. Rzeuski, "QT Interval Prolongation and Tosarde de Pointes Due to a Coadministeration of Ciprofloxacin and Azimilide in a Patient with Implantable Cardioverter Defibrillator," Pacing and Clinical Electrophysiology, Vol. 30, No. 8, 2007, pp. 1043-1046. doi:10.1111/j.1540-8159.2007.00809.x

[73] H. Singh, K. Kishore, M. S. Gupta, S. Khetarpals, S. Jain and M. Mangla, "Reported Case of Ciprofloxacin Induced QTC Prolongation," Journal of the Association of Physicians of India, Vol. 50, 2002, pp. 430-431.

[74] A. Keivanidon, C. Arnaontoglou, A. Krommydas, G. Papanikoloan, K. Tsiptses and C. Kirpizidisc, "Ciprofloxacin Induced Acquired Long QT Syndrome in a Patient under Class 111 Antiarrthymic Therapy," Cardiology, Vol. 16, No. 2, 2009, pp. 172-174.

[75] G. J. Noel, "Effects of Three Fluoroquinolone on QT Interval in Healthy Adults after Single Doses," Clinical Pharmacology, Vol. 73, No. 4, 2003, pp. 292-303.

[76] D. Katrisis and A. J. Camm, "Quinolones Cardioprotective or Cardiotoxic," Pacing and Clinical Electrophysiology, Vol. 26, No. 12, 2003, pp. 2317-2320.

[77] D. Altman, U. Eggmann and P. Amman, "Drug Induces QT Prolongation," Wiener Klinische Wochenschrift, Vol. 120 No. 5-6, pp. 128-135.

[78] E. Rubinstein and J. Camm, "Cardiotoxicity of Fluoroquinolones," Journal of Antimicrobial Chemotherapy, Vol. 49, No. 4, 2002, pp. 593-596. doi: $10.1093 / \mathrm{jac} / 49.4 .593$

[79] S. Patenes, F. Marte, G. Di Bella, A. Gurro and S. Coglitore, "QT Interval Prolongation, Torsade de Pointes and Renal Failure," International Journal of Cardiology, Vol. 12, No. 2, 2008, pp. 71-73.

[80] P. B. Iannini and G. S. Tittoson, "Evaluating the Risk of Cardiotoxicity," Pharmacotherapy, Vol. 21, No. 3, 2001, pp. 261-262. doi:10.1592/phco.21.3.261.34200 